\title{
Correction to: Technologies for Therapy and Assistance of Lower Limb \\ Disabilities: Sit to Stand and Walking
}

Isela Carrera, Hector A. Moreno, Sergio Sierra, Alexandre Campos, Marcela Munera, and Carlos A. Cifuentes

\section{Correction to:}

Chapter 4 in: M. Cardona et al., Exoskeleton Robots for Rehabilitation

and Healthcare Devices, SpringerBriefs in Applied Sciences and Technology, https://doi.org/10.1007/978-981-15-4732-4_4

The original version of the chapter was published with incorrect authors list. This has now been corrected. 